\title{
ON THE PENETRATION OF THE 660 KM PHASE CHANGE BY MANTLE DOWNFLOWS
}

\author{
David Bercovici ${ }^{1}$, Gerald Schubert ${ }^{2}$, and Paul J. Tackley ${ }^{3}$
}

\begin{abstract}
We present a simple analytic model of the interaction of cold convective downwelling currents with an endothermic phase change. The model describes the ponding and lateral spreading of downflows along the phase transition interface. A simple comparison of the vertical forces on the ponding material provides a necessary condition for a downflow to penetrate the phase boundary. This condition is fundamentally dependent on the geometry of the downflow. For planar downwellings, the model predicts a minimum ponding time before the structure can penetrate the phase boundary. For columnar (axisymmetric) downflows, there is no minimum time of spreading required before penetration can proceed. The model thus provides an explanation for the observation that in numerical models of three-dimensional convection with an endothermic phase change, cylindrical downflows penetrate the phase interface while planar ones do not. Since descending slabs in the Earth's mantle display a wide spectrum of geometries between planar and cylindrical (given various trench curvatures, as well as intersections of two or more subduction zones), this phenomenon may explain, in part, why some slabs appear to extend into the lower mantle while others are deflected at the $660 \mathrm{~km}$ discontinuity.
\end{abstract}

\section{Introduction}

It is now generally accepted that the seismic discontinuity at $660 \mathrm{~km}$ depth in the mantle [Shearer, 1991; Shearer and Masters, 1992] is associated with the phase transition from spinel to perovskite plus magnesiowüstite. The phase change is an endothermic one with a Clapeyron slope of -2 to $-6 \mathrm{MPa} \mathrm{K}^{-1}$ [Ito and Takahashi, 1989; Ito et al., 1990]. The penetrability of this boundary by mantle flow has been the subject of debate for over twenty years [e.g. Silver et al., 1988]. Evidence of such penetration by subducting slabs was first suggested by studies of seismic traveltime anomalies through slabs [e.g., Creager and Jordan, 1986]. More recently, however, tomographic images of the Earth's mantle indicate that slab penetration is not universal. Some descending slabs (e.g., those associated with the Java, Kuril-Kamchatka and Mariana trenches) appear to penetrate the phase transition while other slabs (e.g., ones connected to the Kurile, Japan and Izu-Bonin trenches) appear to deflect along the boundary [van der Hilst et al.,

\section{Dept. of Geology \& Geophysics, University of Hawaii} 2 Dept. of Earth \& Space Sciences, University of California 3 Seismological Lab., California Institute of Technology

\section{Copyright 1993 by the American Geophysical Union.}

1991; Fukao et al., 1992]. West of the southern Kuril and Izu-Bonin arcs is a $1000 \mathrm{~km}$ long depression in the 660 $\mathrm{km}$ interface which is consistent with the horizontal deflection of the subducting slab by this boundary [Shearer and Masters, 1992].

Recent numerical simulations of mantle convection which incorporate the thermal and dynamical effects of an endothermic phase transition [Machetel and Weber, 1991; Peltier and Solheim, 1992; Zhao et al., 1992; Tackley et al., 1993; Weinstein, 1993; Honda et al., 1993a; Steinbach et al., 1993] have begun to shed light on the interaction of mantle flow - in particular mantle downwellings or idealized slabs - and the $660 \mathrm{~km}$ transition. In the numerical models, descending currents impinge on the phase boundary and their cold temperature anomaly deflects the phase transition downward. The hydrostatic head from this deflection results in an upward force which resists the passage of the downwelling through the phase change. Cold material piles up and spreads laterally on the phasechange boundary. Eventually, enough cold material accumulates to overcome the upward buoyancy force of the phase transition and the cold material pours through the phase boundary in what has been termed an 'avalanche' or 'flushing' event [Tackley et al., 1993; Honda el al., 1993a]. In two-dimensional numerical models [both planar and axisymmetric, Zhao et al., 1992; Machetel and Weber, 1991; Peltier and Solheim, 1992; Weinstein, 1993; Steinbach et al., 1993], there are relatively few avalanche events separated by long periods of time in which convection occurs in a two-layer mode, i.e., the horizontal phase change boundary divides the system into separately convecting layers. The system is in a one-layer or whole-layer mode of convection for a relatively short period of time during an avalanche event. However, in three-dimensional numerical models [Tackley et al., 1993; Honda et al., 1993a,b], flushing events occur mainly as quasi-cylindrical downflows at the intersections of downwelling sheets and are more numerous and frequent, if not ubiquitous. Convection in these models is essentially always in a whole-layer mode, though its spatial characteristics are largely controlled by the inhibitory nature of the endothermic phase change.

The three-dimensional numerical results therefore suggest that the penetration of the phase change is significantly determined by the geometry of the cold descending currents or slabs. In this paper we explore this suggestion with a simple analytic theory. The theory examines the ponding of cold downflows at an endothermic phase boundary, and the conditions by which these ponds can eventually break through the boundary. We seek to gain basic insight into how slab or downflow geometry controls the penetration of the phase transition; in particular, we address the question of why, in the three-dimensional models, cold material pours through the phase change in cylindrical forms, while downwelling sheets do not penetrate the phase change at all. 
Theory

When a cold viscous downwelling impinges on an endothermic phase-change interface, we assume it first pools, or spreads laterally as a viscous gravity current. This is shown to occur in numerical models of isoviscous convection, though the theory to follow is most applicable if the downwelling is of much higher viscosity than the overlying mantle (and is thus more consistent with slab-mantle viscosity contrasts). The downward force this current exerts on the interface is simply its weight excess (relative to its surroundings). If the interface is an endothermic phase change, it will be deflected downward by the cold temperature anomaly of the slab material; the negative hydrostatic head due to this deflection exerts an upward force on the pooling slab material. By comparing the downward force of the cold spreading gravity current to the upward force of the deflected boundary, we can derive a necessary condition for the downflow to penetrate an endothermic phase change. Once the downward force of the gravity current exceeds the upward force of the boundary deflection, penetration can occur. However, this is only a necessary condition for penetration and does not imply that penetration will immediately proceed. Initiation of penetration depends on several other processes, e.g., the viscous resistance of the lower layer, and diffusion of the downwelling's temperature anomaly. In the following analysis we examine this necessary condition for two-dimensional and cylindrically symmetric downflows to provide insight into how the geometry of a descending current influences its ability to penetrate the phase boundary.

The horizontally averaged downward force per unit area of a gravity current of thickness $h$ on a phase-change interface is $\rho \alpha \Delta T g \bar{h}$ where $\rho$ is the upper mantle density, $T-\Delta T$ is the temperature of the current ( $T$ being the ambient mantle temperature), $\alpha$ is thermal expansivity, $g$ is gravity and the over-bar indicates a horizontally averaged quantity (see Figure 1). The average downward deflection of the endothermic phase change by the current's temperature anomaly is $\bar{\delta}=\frac{\gamma \Delta T}{\rho g}$ where $\gamma$ is the magnitude of the Clapeyron slope of the phase transition (with units of $\mathrm{Pa}$ $\mathrm{K}^{-1}$ ) and the temperature of the phase boundary beneath the gravity current is assumed to be fixed at $T-\Delta T$. The average upward pressure on the base of the gravity current is then $\gamma \Delta T \Delta \rho / \rho$ where $\Delta \rho$ (assumed positive) is the den- sity contrast between the the two phases. The ratio of the downward to upward force is

$$
F_{R}=\frac{\rho^{2} \alpha g \bar{h}}{\gamma \Delta \rho} .
$$

The force ratio $F_{R}$ is thus independent of the magnitude of the downflow's temperature anomaly. The quantity $F_{R}^{-1}$ is similar to the phase change density parameter $S$ of Schubert and Turcotte (1971) and the phase change buoyancy parameter $P$ of Christensen and Yuen (1985) (with $\bar{h}$ replacing the characteristic layer depth). In this paper, we seek conditions for which the ratio $F_{R}$ exceeds unity.

A planar downflow impinging on the interface would ideally spread as a two-dimensional gravity current. The mean thickness of such a current supplied by a constant volumetric flow rate per unit length $q$ (with units of $\mathrm{m}^{2} \mathrm{~s}^{-1}$ ), is

$$
\bar{h}=\frac{1}{x_{N}} \int_{0}^{x_{N}} h d x=\frac{q t}{x_{N}}
$$

where $x$ is the distance from the center of the symmetrically spreading current. The leading edge of the current propagates outward in time $t$ and occurs at [Huppert, 1982]

$$
x_{N}=\eta_{N}\left[\frac{\alpha \Delta T g q^{3} t^{4}}{3 \nu}\right]^{1 / 5}
$$

where $\nu$ is the kinematic viscosity of the gravity current (and slab or downwelling) and $\eta_{N}$ is a dimensionless constant approximately equal to 1 .

A cylindrically axisymmetric downflow impinging on the phase change interface would ideally spread as a diskshaped gravity current. The average thickness of an axisymmetric gravity current supplied by a constant volumetric flow rate $Q$ (units of $\mathrm{m}^{3} \mathrm{~s}^{-1}$ ) is [Huppert, 1982]

$$
\bar{h}=\frac{2 \pi}{\pi r_{N}^{2}} \int_{0}^{r_{N}} h r d r=\frac{Q t}{\pi r_{N}^{2}}
$$

where $r$ is the radius from the center of the current and the leading edge of the current occurs at

$$
r_{N}=\xi_{N}\left[\frac{\alpha \Delta T g Q^{3} t^{4}}{3 \nu}\right]^{1 / 8}
$$

where $\xi_{N}$ is a dimensionless constant approximately equal to $3 / 4$.

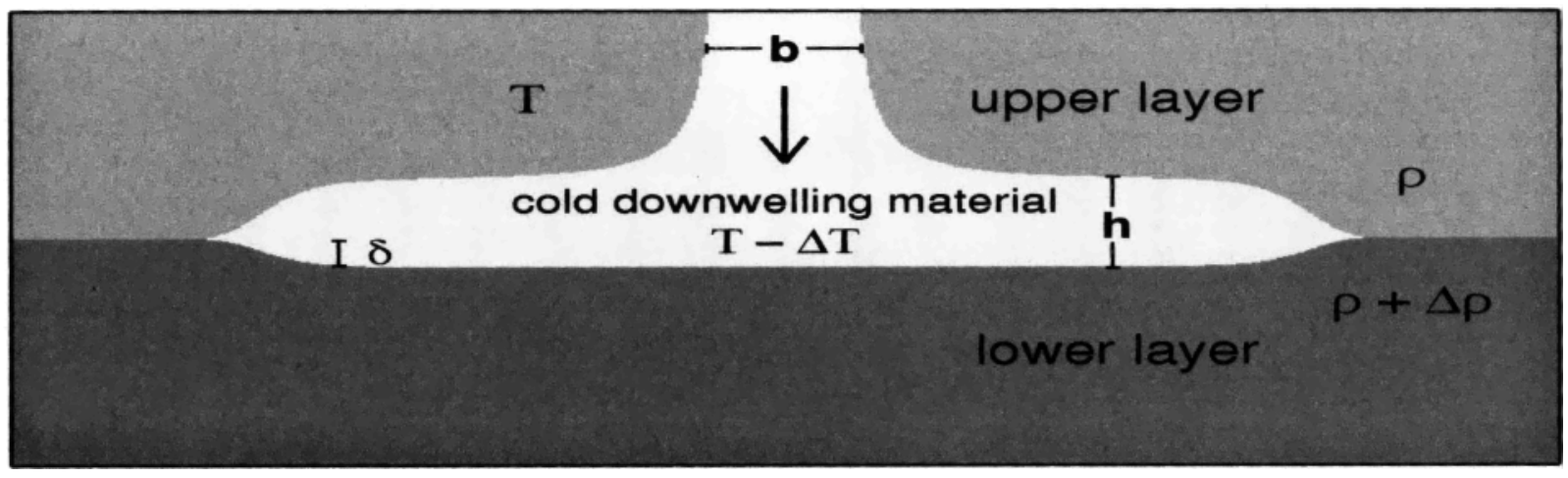

Fig. 1. Sketch of a cold descending current ponding and spreading laterally along an endothermic phase boundary. The downflow and gravitationally spreading lens is either planar (two-dimensional Cartesian) or columnar (cylindrically axisymmetric). See text for definition of symbols. 
The force ratio $F_{R P}$ for the two-dimensional planar current is given by

$$
F_{R P}^{5}=\left[\frac{\rho^{2}}{\gamma \Delta \rho \eta_{N}}\right]^{5}(g \alpha)^{4} \frac{3 \nu q^{2} t}{\Delta T}
$$

while the ratio for the cylindrical current $F_{R C}$ is given by

$$
F_{R C}^{4}=\left[\frac{\rho^{2}}{\gamma \Delta \rho \pi \xi_{N}^{2}}\right]^{4}(g \alpha)^{3} \frac{3 \nu Q}{\Delta T}
$$

For a planar downwelling, the ratio $F_{R P}$ depends on time and is thus bound to exceed unity for $t$ sufficiently large. The required time allows the gravity current to inflate vertically until it has sufficient weight to penetrate the phase boundary. For the columnar downwelling, the spreading gravity current does not inflate similarly since its lateral growth exactly balances the injection of fluid at its center. The ratio $F_{R C}$ is therefore independent of time and hence either exceeds unity at all times, or never exceeds unity.

Several strengths and limitations of the model are worth enumerating. The gravity-current similarity theory employed here [Huppert, 1982] is most accurate if the current is thin and/or the upper surface of the current is essentially free-slip. Though we cannot assume the former criterion, the latter one is valid for a current whose viscosity is much higher than that of the overlying medium, as in this paper's model. However, the underlying boundary (e.g., the phase-change interface) is assumed rigid, which is perhaps less appropriate for applications to the Earth since the lower mantle is not likely to be much more viscous than a slab. Even so, the major drag on the spreading slab is likely to come from the lower mantle; thus, short of rigorously solving for lower-mantle flow (which is unwarranted in this simple theory), use of a rigid boundary is more appropriate than a free-slip one. Allowance for a mobile lower layer would cause the gravity current to spread faster, leading to a smaller $\bar{h}$ at a given time; some simple analysis, however, suggests that the dependence of $F_{R}$ on time is unlikely to change significantly. The gravity-current similarity theory also does not account for deflection of the lower boundary. The theory is thus most applicable if $h>\delta$ (or $h \rho g /(\gamma \Delta T)>1$ ) which is valid for typical mantle parameters (see the "Applications" section below for parameter values). The similarity theory also assumes that fluid is injected at a constant volumetric flow rate from an infinitesimally thin source (e.g., a zero-thickness downwelling). Finally, thermal diffusion of the downflow's temperature anomaly is neglected. The decrease in $\Delta T$ with time causes two competing effects. First, it reduces the relative density of the gravity current, compelling it to spread more slowly. Second, the decrease in viscosity due to warming of the gravity current induces the spreading rate to increase. Given the typical temperature-dependent viscosities of silicates, the latter effect is probably more influential. Even with these considerations, the typical diffusion time-scale for a $100 \mathrm{~km}$ thick downflow with thermal diffusivity $\kappa=10^{-6} \mathrm{~m}^{2} \mathrm{~s}^{-1}$ is approximately $0.3 \mathrm{Gyr}$; as shown below, this is significantly longer than the time necessary for $F_{R}$ to reach unity. Thus, it is likely that if a force balance favorable to phase boundary penetration is achieved, it will occur before diffusion becomes significant.

\section{Application}

With application to the real mantle and models thereof in mind, we evaluate the above force ratios for mantle-type parameter values. We assume that the thickness of a planar downflow is $b=100 \mathrm{~km}$, similar to that of a descending slab in the mantle. For a conservative descent velocity $w=10 \mathrm{~mm} \mathrm{yr}^{-1}$, the volumetric flux per unit length is $q=b w=10^{3} \mathrm{~m}^{2} \mathrm{yr}^{-1}$. A columnar downflow approximates either a strongly curved sheet-like downwelling or slab (e.g., slabs associated with highly arcuate trenches), or the intersection of several planar downwellings. A tightly curled downwelling of thickness $b$ is analogous to a column of radius $b$ for which $Q=\pi b^{2} w=\pi q b$. For the intersection of planar downflows, we assume the volumetric flux of each current adds at the intersection; this is suggested by studies of three-dimensional convection which show that intersections of planar currents are velocity maxima [Bercovici et al., 1989a, 1992]. In this case, we assume for simplicity that $Q=n q b$ where $n$ is the number of intersecting downflows. In three-dimensional convection, downwelling sheet intersections are at the very least triple junctions [Houseman, 1988; Bercovici, 1989b; Tackley et al., 1993], implying $n \geq 3$. Thus regardless of whether columnar downwellings simulate strongly curved or intersecting downflows (or slabs), the ratio $\frac{Q}{a b}$ is typically $\geq 3$.

The dynamic viscosity of the downflow is $\nu \rho=2 \times$ $10^{24} \mathrm{~Pa} \mathrm{~s}$, its temperature anomaly is $\Delta T=300 K$, density is $\rho=4 \times 10^{3} \mathrm{~kg} \mathrm{~m}^{-3}$, and for the remaining parameters we use $\alpha=3 \times 10^{-5} \mathrm{~K}^{-1}, \gamma=4 \mathrm{MPa} \mathrm{K}^{-1}$, and $\Delta \rho / \rho=10^{-1}$. We find that $F_{R C}^{4}=1.3 \frac{Q}{3 q b}$, while $F_{R P}^{5}=1.3 \times 10^{-7} t$ where $t$ is in years. Thus, $F_{R C}$ is likely to just exceed unity, given that $\frac{Q}{q b}$ is $\geq 3$; the downward force of the cylindrical downflow can thus overcome the upward force of the deflected phase boundary as soon as the cylindrical downflow impinges on the boundary. Alternatively, $F_{R P}$ will only exceed unity for $t>8 \mathrm{Ma}$. A planar slab will be necessarily delayed at the phase boundary at least ten million years. It should be emphasized that this is only the time necessary for a force balance favorable to penetetration to be attained. The actual initiation of penetration depends on other processes such as viscous resistance from the lower mantle, and thermal diffusion of the downflow's temperature anomaly; penetration is thus likely to occur well after $F_{R}$ exceeds unity. With all its intrinsic simplifications, the above analysis merely suggests that columnar downwellings can begin to initiate penetration upon contact with the phase boundary; whether actual penetration thenceforth proceeds is not predicted. In contrast, the planar downflow will be delayed some finite amount of time before it has even deposited enough weight on the boundary to initiate penetration.

\section{Relevance to Convection Models and Subducting Slabs}

The above theory implies that the cylindrical structures at the intersections of a downwelling network in three-dimensional convection models will penetrate into the lower layer first and begin to funnel off downwelling 
fluid from the adjacent downwelling sheets. Once this occurs, the volume flux $q$ into the two-dimensional gravity currents will diminish, causing an even longer - if not indefinite - delay time. In other words, cylindrical penetrations into the lower layer sap off fluid from the planar downflows thereby inhibiting them from descending into the lower layer. This effectively explains the observation from numericál módels that only cylindricál downwellings penetrate the endothermic phase boundary.

The theory of this paper might also provide insights into the apparent differences in the penetrability of the $660 \mathrm{~km}$ transition by descending slabs. The basic prediction of the analytic model presented here, as well as the three-dimensional numerical models, is that cylindrical-like downflows are the preferred sites of phase change penetration. These models are, of course, greatly simplified and do not incorporate some very important aspects of slab dynamics, perhaps most importantly the effects of oblique subduction (e.g., Kincaid and Olson, 1987). However, if applicable to the Earth, they predict relatively easier penetration of the $660 \mathrm{~km}$ transition by strongly curved slabs (e.g., the slab connected to South Sandwich trench), or by slab material associated with the intersection of two or more trenches (e.g., the triple junction of the Izu-Bonin, Japan and Ryuku-Nansai trenches, and the junction of the Aleutian and Kurile trenches). The test of this prediction, however, necessarily awaits future seismic studies.

Acknowledgments. The theory presented here was conceived during a visit by G.S. to the University of Hawaii under the SOEST Dean's Visiting Scholar Program. This work was supported by NASA under grants NAGW-2646 to G.S. and NAGW-3015 to D.B.

\section{References}

Bercovici, D., G. Schubert, G. A. Glatzmaier, and A. Zebib, Three-dimensional thermal convection in a spherical shell, J. Fluid Mech. 206, 75-104, 1989a.

Bercovici, D., G. Schubert and G. A. Glatzmaier, Threedimensional, spherical models of convection in the Earth's mantle, Science 244, 950-955, 1989b.

Bercovici, D., G. Schubert and G. A. Glatzmaier, Threedimensional, infinite Prandtl number, compressible convection in a basally heated spherical shell, J. Fluid Mech. 239, 683-719, 1992.

Christensen, U. R., and D. A. Yuen, Layered convection induced by phase transitions, J. Geophys. Res. 90, 10,29110,300, 1985.

Creager, K.C. and T.H. Jordan, Slab penetration into the lower mantle beneath the Mariana and other island arcs of the northwest Pacific, J. Geophys. Res. 91, 3573-3589, 1986.

Fukao, Y., M. Obayashi, H. Inoue, and M. Nenbai, Subducting slabs stagnant in the mantle transition zone, $J$. Geophys. Res. 97, 4809-4822, 1992.

Honda, S., S. Balachandar, D. A. Yuen, and D. Reuteler, Three-dimensional mantle dynamics with an endothermic phase transition, Geophys. Res. Lett. 20, 221-224, 1993a.

Honda, S., D. A. Yuen, S. Balachandar, and D. Reuteler, Three-dimensional instabilities of mantle convection with multiple phase transitions, Science 259, 1308-1311, $1993 \mathrm{~b}$.
Houseman, G. The dependence of convection planform on mode of heating, Nature 332, 346-349, 1988.

Huppert, $H$. The propagation of two-dimensional and axisymmetric viscous gravity currents over a rigid horizontal surface, J. Fluid Mech. 121, 43-58, 1982.

Ito, E., and E. Takahashi, Postspinel transformation in the system $\mathrm{Mg}_{2} \mathrm{SiO}_{4}-\mathrm{Fe}_{2} \mathrm{SiO}_{4}$ and some geophysical implications, J. Geophys. Res. 94, 10,637-10,646, 1989.

Ito, E., M. Akaogi, L. Topor, and A. Navrotsky, Negative pressure-temperature slopes for reactions forming $\mathrm{MgSiO}_{3}$ perovskite from calorimetry, Science 249, 1275$1278,1990$.

Kincaid, C., and P. L. Olson, An experimental study of subduction and slab migration, J. Geophys. Res. 92, 13,83213,840, 1987.

Machetel, P., and P. Weber, Intermittent layered convection in a model with an endothermic phase change at 670 km, Nature 350, 55-57, 1991.

Peltier, W. R., and L. P. Solheim, Mantle phase transitions and layered chaotic convection, Geophys. Res. Lett. 19, 321-324, 1992.

Schubert, G., and D. L. Turcotte, Phase changes and mantle convection, J. Geophys. Res. 76, 1424-1432, 1971.

Shearer, P. M., Constraints on upper mantle discontinuities from observations of long-period reflected and converted phases, J. Geophys. Res. 96, 18,147-18,182, 1991.

Shearer, P. M., and T. G. Masters, Global mapping of topography on the $660-\mathrm{km}$ discontinuity, Nature 355,791 796, 1992.

Silver, P. G., R. W. Carlson, and P. Olson, Deep slabs, geochemical heterogeneity, and the large-scale structure of mantle convection: investigation of an enduring paradox, Ann. Rev. Earth Planet. Sci. 16, 477-541, 1988.

Steinbach, V., D. A. Yuen, and W. Zhao, Instabilities from phase transitions and the timescales of mantle thermal evolution, Geophys. Res. Lett. 20, 1119-1122, 1993.

Tackley, P. J., D. J. Stevenson, G. A. Glatzmaier, and G. Schubert, Effects of an endothermic phase transition at $670 \mathrm{~km}$ depth in a spherical model of convection in the Earth's mantle, Nature 361, 699-704, 1993.

van der Hilst, R. D., R. Engdahl, W. Spakman, and G. Nolet, Tomographic imaging of subducted lithosphere below northwest Pacific island arcs, Nature 353, 37-43, 1991.

Weinstein, S. A., Catastrophic overturn of the Earth's mantle driven by multiple phase changes and internal heat generation, Geophys. Res. Lett. 20, 101-104, 1993.

Zhao, W., D. A. Yuen, and S. Honda, Multiple phase transitions and the style of mantle convection, Phys. Earth Planet. Int. 72, 185-210, 1992.

D. Bercovici, Department of Geology \& Geophysics, School of Ocean \& Earth Science \& Technology, University of Hawaii, Honolulu, HI 96822.

G. Schubert, Department of Earth \& Space Sciences, University of California, Los Angeles, CA 90024

P.J. Tackley, Seismological Laboratory, California Institute of Technology, Pasadena, CA 91125

(Received August 10, 1993; accepted September 20, 1993.) 